\title{
UV laser-induced poling inhibition in lithium niobate crystals
}

\author{
Y. J. Ying ${ }^{1}$, C. L. Sones ${ }^{1}$, H. Steigerwald ${ }^{2}$, F. Johann ${ }^{2}$ E. Soergel ${ }^{2}$, K. Buse ${ }^{2}$, R. W. Eason ${ }^{1}$, and S. Mailis ${ }^{1}$ \\ 1. Optoelectronics Research Centre, University of Southampton, Southampton, SO17 1BJ, U.K. \\ 2. Institute of Physics, University of Bonn, Wegelerstrasse 8, 53115 Bonn, Germany
}

The development of methods for ferroelectric domain engineering in lithium niobate (LN) is of special importance as it not only enables the fabrication of efficient non-linear devices (PPLN) [1] but also constitutes a very effective path towards micro-structuring of this important ferroelectric crystal [2].

UV laser-induced poling inhibition is a process whereby domain inversion is prohibited as a result of previous UV laser irradiation of the crystal. The effect was first demonstrated using a frequency-doubled argon ion laser $(244 \mathrm{~nm})$ to irradiate the $+\mathrm{z}$ face of congruently melting undoped and $\mathrm{MgO}$-doped $\mathrm{LN}$ single crystals followed by electric field poling [3]. In that initial report the potential of the method for both, ferroelectric domain engineering and micro-structuring, was demonstrated.

In this contribution we will present an investigation of the characteristics of these poling-inhibited (PI) domains. The PI domain depth, width, and quality were studied, by chemical etching and piezoresponse force microscopy for a range of fabrication conditions such as the intensity, fluence/wavelength of the illuminating UV laser, and conditioning of the crystal. For this reason LN samples were exposed to focused UV laser light ( $4 \mu \mathrm{m}$ focal spot diameter) from a tunable argon-ion laser which could provide a range of wavelengths from $275 \mathrm{~nm}$ to $305 \mathrm{~nm}$. UV irradiated linear tracks were produced by scanning the crystal sample in front of the focused laser beam at constant velocity. The LN samples were subsequently poled, by the application of an external electric field across the opposite polar faces at room temperature, resulting in PI domains at the UV irradiated areas.

In order to investigate the depth profile of the PI domains the samples were chemically etched in HF acid after being wedge polished at the shallow angle of $5^{\circ}$. This increased the domain-depth-measurement resolution by one order of magnitude $\left(1 / \sin 5^{\circ}\right)$ as compared to the commonly used y-face polishing/etching method.
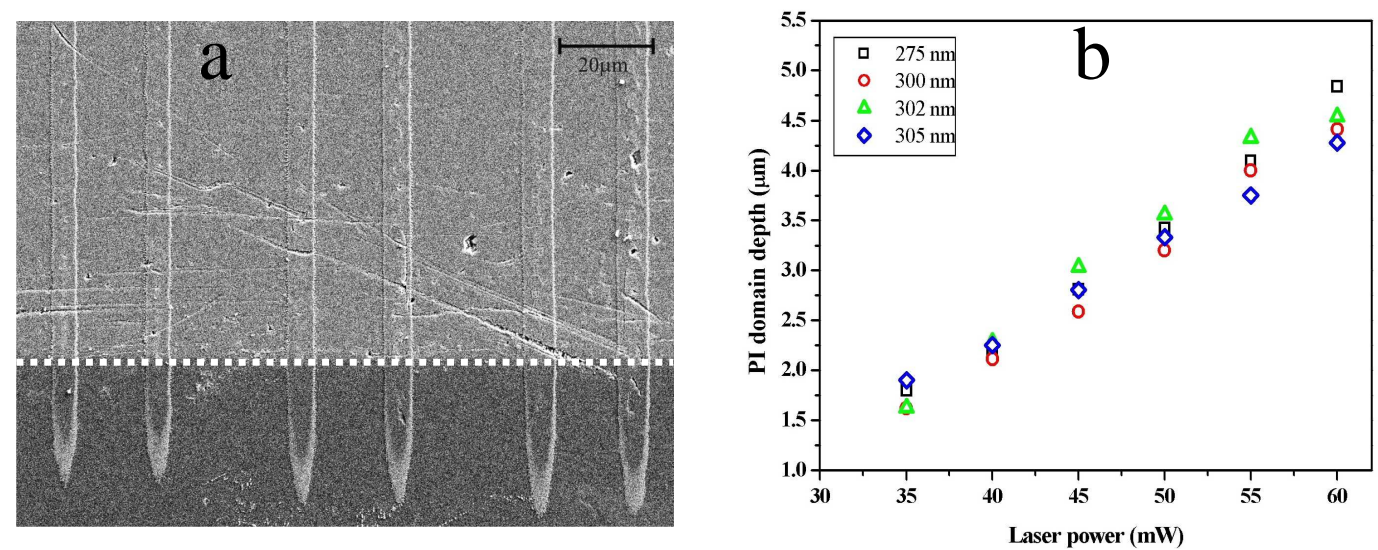

Fig. 1 a) SEM image of the wedge-polished and etched sample showing PI domain tracks. The dashed line indicates the change of slope due to wedge polishing and b) plot of PI domain depth as a function of laser power for different illuminating laser wavelengths; black square, red circle, green triangle, and blue diamond correspond to 275, 300, 302 and $305 \mathrm{~nm}$ laser illumination respectively. The scanning speed was $1 \mathrm{~mm} \mathrm{~s}^{-1}$.

Figure 1a shows an SEM image of the PI domains revealed after HF etching of a wedge-polished sample. The slight contrast difference in the SEM image indicates the change in slope due to the wedge. For clarity the change of slope is also indicated by the dashed line. Each pair of tracks corresponds to different fabrication conditions, in this case different scan speeds, resulting in domains of different depth. The depth of PI domains as a function of laser power for different illuminating laser wavelengths is shown in Fig. 1b. It is seen that the depth of the PI domains increases with increasing laser power as expected but interestingly is not very sensitive to the wavelength of the illuminating laser. However, in all cases the PI domain depths are suitable for both, nonlinear optical waveguide applications and surface micro-structuring.

\section{References}

[1] M. Yamada, N. Nada, M. Saitoh, and K. Watanabe Appl. Phys. Lett. 62, 435 (1993)

[2] I. E. Barry, G. W. Ross, P. G. R. Smith, R. W. Eason, and G. Cook, Mat. Lett. 37, 246 (1998)

[3] C. L. Sones, A. C. Muir, Y. J. Ying, S. Mailis, R. W. Eason, T. Jungk, A. Hoffmann, and E. Soergel, Appl. Phys. Lett. 92, 072905 (2008) 